\title{
Comparison of Statistical Estimation Techniques for Mars Entry, Descent, and Landing Reconstruction from MEDLI- like Data Sources
}

\author{
Soumyo Dutta, ${ }^{1}$ Robert D. Braun, ${ }^{2}$ Ryan P. Russell, ${ }^{3}$ Ian G. Clark ${ }^{4}$ \\ Georgia Institute of Technology, Atlanta, GA 30332-0150, USA \\ and \\ Scott A. Striepe \\ NASA Langley Research Center, Hampton, VA 23681-0001, USA
}

\begin{abstract}
Flight data from an entry, descent, and landing (EDL) sequence can be used to reconstruct the vehicle's trajectory, aerodynamic coefficients and the atmospheric profile experienced by the vehicle. Past Mars missions have contained instruments that do not provide direct measurement of the freestream atmospheric conditions. Thus, the uncertainties in the atmospheric reconstruction and the aerodynamic database knowledge could not be separated. The upcoming Mars Science Laboratory (MSL) will take measurements of the pressure distribution on the aeroshell forebody during entry and will allow freestream atmospheric conditions to be partially observable. This data provides a mean to separate atmospheric and aerodynamic uncertainties and is part of the MSL EDL Instrumentation (MEDLI) project. Methods to estimate the flight performance statistically using on-board measurements are demonstrated here through the use of simulated Mars data. Different statistical estimators are used to demonstrate which estimator best quantifies the uncertainties in the flight parameters. The techniques demonstrated herein are planned for application to the MSL flight dataset after the spacecraft lands on Mars in August 2012.
\end{abstract}

\section{Nomenclature}

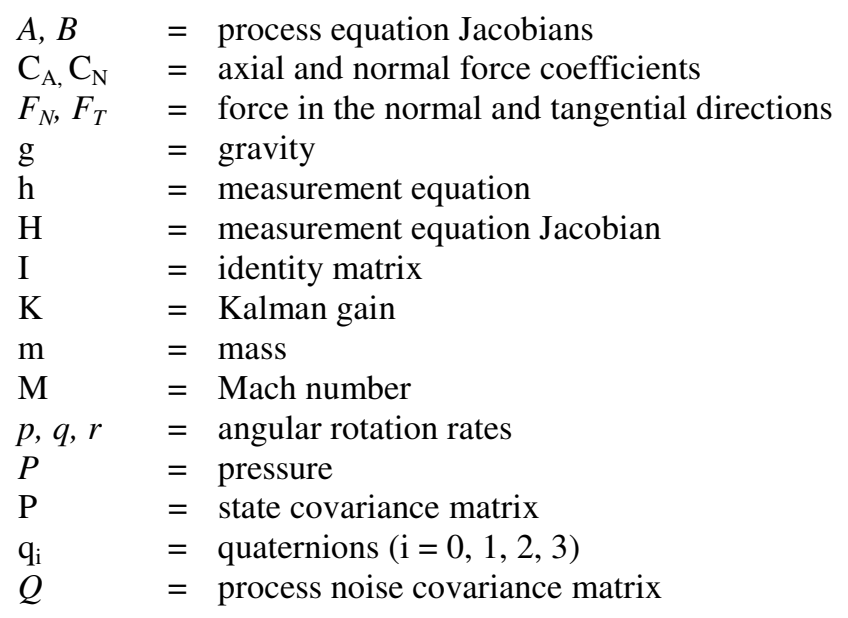

\footnotetext{
${ }^{1}$ Graduate Research Assistant, Daniel Guggenheim School of Aerospace Engineering, AIAA Student Member.

${ }^{2}$ David and Andrew Lewis Professor of Space Technology, Daniel Guggenheim School of Aerospace Engineering, AIAA Fellow.

${ }^{3}$ Assistant Professor, Daniel Guggenheim School of Aerospace Engineering, AIAA Senior Member.

${ }_{5}^{4}$ Visiting Assistant Professor, Daniel Guggenheim School of Aerospace Engineering, AIAA Member.

${ }^{5}$ Aerospace Engineer, Atmospheric Flight and Entry Systems Branch.
} 


$\begin{array}{ll}r & =\text { planet-centric radius } \\ R & =\text { measurement covariance matrix } \\ u, v, w & =\text { velocity in body axis } \\ V & =\text { velocity } \\ W & =\text { sigma points weighting factors } \\ x & =\text { state vector } \\ \mathrm{y} & =\text { measurement } \\ \alpha & =\text { angle of attack } \\ \beta & =\text { sideslip angle } \\ \gamma & =\text { flight-path angle } \\ \theta & =\text { longitude } \\ \lambda, \alpha, \beta, \kappa & =\text { UKF tuning parameters } \\ \rho & =\text { density } \\ \sigma & =\text { standard deviation } \\ v & =\text { bank angle } \\ \phi & =\text { planet-centric latitude } \\ \psi & =\text { heading angle } \\ \omega & =\text { planet rotation rate }\end{array}$

\section{Introduction}

CINCE Viking 1 and 2 landed on Mars in 1976, the United States has successfully sent four other spacecraft to the Martian surface. However, failures have also accompanied the exploration of Mars, such as the 1999 Mars Polar lander mission that lost contact during EDL. Mitigating the large uncertainties that exist in engineering models used during Mars EDL design may reduce design conservatism and EDL system mass while avoiding some of the problems of these previous missions.

Trajectory, atmosphere and aerodynamic coefficient reconstructions from flight data allow quantification of the uncertainties in the vehicle performance and the Martian environment. Most of the past reconstructions of Mars vehicle data have been conducted largely using a deterministic methodology, ${ }^{1-5}$ where the flight data, such as onboard sensed accelerations and angular rates, were used to reconstruct the trajectory without explicitly considering uncertainties in the measurement value or in the flight dynamics. Unfortunately, under such a situation, off-nominal data could lead to the divergence of the estimate of the trajectory. The deviation in the trajectory estimation can spread to the reconstruction of other performance parameters.

Moreover, atmosphere reconstruction efforts for the past missions have lacked direct measurement of the freestream atmospheric conditions. Thus, the atmospheric reconstruction has been based on the on-board inertial measurement unit (IMU) data, deterministic reconstruction of velocity and an assumption of perfect knowledge of the aerodynamic coefficients. ${ }^{6}$ This assumption has led to the confounding of the atmospheric and aerodynamic coefficient uncertainties. However, the upcoming MSL mission will include instruments that will provide a direct measurement of the pressure distribution over the entry body's aeroshell during EDL. The pressure data can be blended with the information from the IMU and the on-board radar altimeter to produce a simultaneous reconstruction of trajectory, atmosphere and aerodynamic parameters. Hence, there is a need to demonstrate a comprehensive methodology that can reconstruct EDL trajectory and other performance characteristics while utilizing information about the uncertainty in the data.

This study demonstrates a statistical estimation process that incorporates all on-board data types in the reconstruction. Datasets with random noise are created from simulated Mars EDL trajectories and then the estimator is used to reconstruct the original EDL parameters.

\section{Mars EDL Reconstruction Background}

A major objective for the reconstruction of flight data is to verify the performance of the vehicle and quantify any off-nominal behavior. Additionally, it is beneficial to consider methods that can quantify uncertainty in the estimated EDL parameters so that they could be used in the Mars EDL design process. Looking at the work of Striepe et al., ${ }^{7}$ who performed design analysis of the MSL mission, one sees a long list of design parameters with their associated uncertainties. Uncertainties exist in the entry conditions (such as flight path angle), center of mass location of the vehicle and sensor biases that will affect the deployment of a stage in the mission. However, the two major sources of uncertainties lie in the knowledge of the aerodynamic coefficients of the vehicle and the atmospheric profile it will encounter. Previous EDL reconstructions have attempted to quantify these uncertainties. 


\section{A. Past Reconstructions}

\section{Deterministic Trajectory Reconstructions}

Table 1 summarizes the various measurements taken during the EDL phase by past and upcoming U.S. missions. Due to the data taken by the past Mars EDL missions, the reconstruction techniques have been mostly limited to deterministic estimation methods. These estimation techniques are similar to the methods used to reconstruct data from strap-down guidance systems. ${ }^{11}$ The procedure is simply to use the inertial measurements to integrate the nonlinear equations of motion. Results from the deterministic trajectory reconstructions can be found in the literature for Viking 1 and 2, ${ }^{1}$ Mars Pathfinder, ${ }^{2}$ Mars Exploration Rovers (MER) ${ }^{3}$ and the Phoenix lander. ${ }^{4,5}$ Unfortunately, these reconstructions do not take into account measurement and process uncertainties and lack the ability to quantify the uncertainty of the estimated parameters.

Table 1. EDL-related measurements taken by U.S. Martian missions.

\begin{tabular}{lccccc}
\hline \hline Measurements & Vikings $^{1,12}$ & Pathfinder $^{2,13,14}$ & MERs $^{3,15}$ & Phoenix $^{4,5}$ & MSL $^{16}$ \\
\hline Accelerometer & $\mathrm{X}$ & $\mathrm{X}$ & $\mathrm{X}$ & $\mathrm{X}$ & $\mathrm{X}$ \\
Three-axis gyroscope & $\mathrm{X}$ & & $\mathrm{X}$ & $\mathrm{X}$ & $\mathrm{X}$ \\
Radar altimeter & $\mathrm{X}$ & $\mathrm{X}$ & $\mathrm{X}$ & $\mathrm{X}$ & $\mathrm{X}$ \\
Thermal protection system recession & & & & $\mathrm{X}$ \\
Pressure (during-EDL) & $\mathrm{X}$ & $\mathrm{X}^{\mathrm{i}}$ & & & $\mathrm{X}$ \\
In-depth temperature (during EDL) & $\mathrm{X}$ & $\mathrm{X}$ & & & $\mathrm{X}$ \\
\hline \hline
\end{tabular}

${ }^{\mathrm{i}}$ Pathfinder only took pressure measurements during subsonic parachute descent

\section{Statistical Trajectory Reconstructions}

There have been some past EDL trajectory reconstruction efforts that have utilized statistical estimation techniques. The method of choice has been the Kalman-Schmidt filter, which is a variant of the more general Kalman filter. Kalman filtering was first utilized for Mars EDL data by Euler et al. ${ }^{12}$ in 1978 to estimate the trajectories for Viking 1 and 2. Euler integrated the equations of motion using the inertial data, and then used the radar altimeter and the terminal landing Doppler data to correct the estimate of the trajectory parameters through a statistical filter. Although the Viking probes sampled the atmosphere during EDL using on-board pressure transducers, Euler's work did not include the pressure measurements within the trajectory estimation procedure; thus, a statistical estimation of both the trajectory and the atmosphere was not conducted at that point.

Linear Kalman filtering was also used by Spencer et al. ${ }^{2}$ in the reconstruction of the Mars Pathfinder data. The tool used measurements, such as radar altimeter data, to correct a nominal trajectory based on the integration of the inertial measurement data. Spencer et al. also utilized a smoothing algorithm to combine trajectory reconstruction from forward and backward runs of the data. However, the Pathfinder reconstruction was conducted using only three degree-of-freedom equations of motion and the study did not report body-fixed angular rates of the vehicle. Hence, calculations of angular parameters involved assumptions about the knowledge of the vehicle's aerodynamics.

\section{Atmosphere Reconstruction}

Another objective of the Mars flight reconstruction is to determine the atmospheric profile encountered by the vehicles during EDL. Without pressure measurements during EDL, density and other atmospheric properties have been estimated deterministically by assuming perfect knowledge of the aerodynamic coefficients and then using the reconstructed velocity of the vehicle and the on-board sensed accelerations to estimate density using equations like the definition of the axial force coefficient. Pressure is then estimated using the hydrostatic equation. Deterministic atmospheric reconstruction procedures were proposed as early as 1965 by Seiff and Reese. ${ }^{17}$ Atmospheric reconstructions of Mars missions using deterministic techniques exist in the literature for the Viking missions, ${ }^{18}$ Mars Pathfinder, ${ }^{2}$ MER $^{15}$ and Phoenix. ${ }^{4}$ As mentioned before, the problem with such estimation is that one confounds atmospheric and aerodynamic uncertainties.

As noted in Table 1, the two Viking missions and Mars Pathfinder had on-board pressure transducers that could be used to estimate the freestream conditions using pressure on the forebody of the vehicle during EDL. However, Pathfinder only took measurements after the parachute deployment ${ }^{13}$ and thus that data cannot be used to reconstruct the atmosphere during the hypersonic EDL phase without additional approximations, such as the hydrostatic equation. Viking 1 and $2,{ }^{18}$ on the other hand, did take pressure measurements even in the hypersonic phase of EDL, but the pressure measurements were not directly used in the trajectory estimation and the uncertainties in the pressure measurements were not included in the estimation algorithm. ${ }^{19}$ 
Non-Mars EDL flight reconstructions have utilized pressure data in statistical estimation procedures. The Shuttle Entry Air Data System (SEADS) program of the 1980's used a flush-mounted air data system to estimate the pressure distribution across the Space Shuttle forebody during entry. ${ }^{20}$ The MEDLI program's pressure data system is in large part based on the SEADS concept. The SEADS project was able reconstruct the freestream conditions during shuttle entry successfully and verified its results with simulation and wind tunnel data. However, reconstructions based on SEADS data did not blend the inertial measurements with the pressure distribution data. Instead, a sequential filter was used in conjunction with a database of pressure distributions on the vehicle forebody to inversely estimate the aerodynamic parameters that could create the pressure measurements at the transducers. Thus, potential coupling between trajectory and atmospheroc uncertainties were not considered by that analysis.

\section{B. Mars Science Laboratory}

Many of the EDL parameter reconstructions in the past have suffered from the lack of direct observations of the freestream conditions and not utilizing measurement uncertainty in the estimation process. However, MSL will carry a set of instruments that will be able to take in-situ measurements of the pressure and temperature distribution on the aeroshell. The instrumentation is known as MEDLI and will consist of the Mars Entry Atmospheric Data System (MEADS) to take pressure measurements and Mars Integrated Sensor Plug (MISP) to take aerothermodynamic data. ${ }^{16}$

MEADS will provide a dataset that will allow the estimation of atmospheric properties without confounding the uncertainties in the knowledge of the aerodynamic coefficients. The science objective of MEADS is to reconstruct the atmospheric properties within certain bounds. Specifically when the dynamic pressure is greater than $850 \mathrm{~Pa}$, the

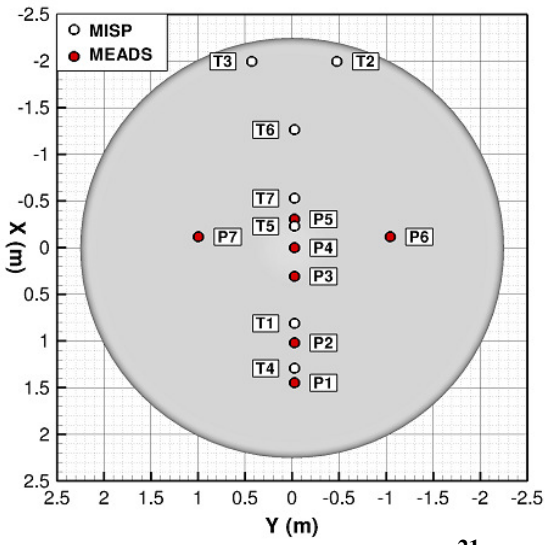

Figure 1. MEDLI sensors. ${ }^{21}$ objective is to estimate freestream Mach number to within \pm 0.1 , dynamic pressure to within $\pm 2 \%$, and angle of attack and sideslip angle to within $\pm 0.5 \mathrm{deg} .{ }^{16}$ In order to achieve these targets, MEADS will collect pressure data from seven pressure transducers located around the forebody of the aeroshell (see Figure 1).

The locations of the transducers are based on the predicted pressure distribution on the aeroshell. The stagnation pressure is expected to be around transducers P1 and P2, while P6 and P7 serve as the transducers which will help reconstruct the sideslip angle. Although the transducers that are used for MEADS can sample at high rates, due to memory constraints, both pressure and temperature data will be saved at an effective sampling rate of $8 \mathrm{~Hz}$. A methodology to utilize MEADS data for reconstruction is described here. Edquist et al. ${ }^{21}$ and Mahzari et al. ${ }^{22}$ describe how MISP data could be used for aerothermodynamic reconstruction.

\section{MEDLI Simulation}

MEDLI-like datasets are simulated in this study to demonstrate the effectiveness of a statistical reconstruction methodology that can incorporate disparate data types and estimate trajectory, atmospheric parameters and aerodynamic coefficients. The Program to Optimize Simulated Trajectories II (POST2) ${ }^{23}$ is used to generate two Mars EDL trajectories which are shown in Figure 2. These trajectories represent the truth data and the reconstructed values are compared with them. There is a nominal EDL trajectory and a dispersed case of the nominal trajectory with perturbations in the vehicle's aerodynamic database, planetary atmosphere and winds.

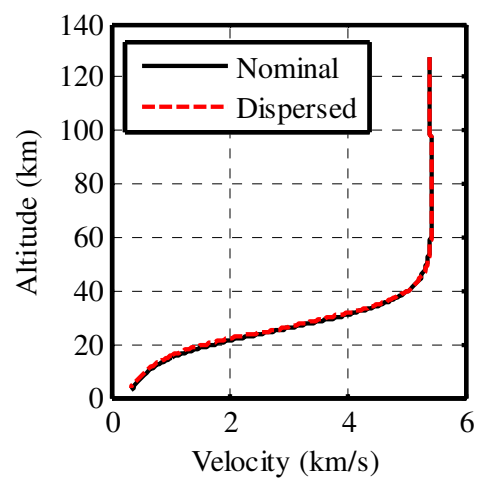

(a) Velocity vs. altitude

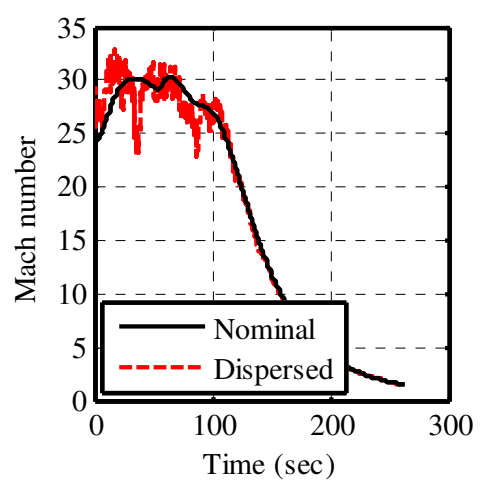

(b) Mach number

4

American Institute of Aeronautics and Astronautics 


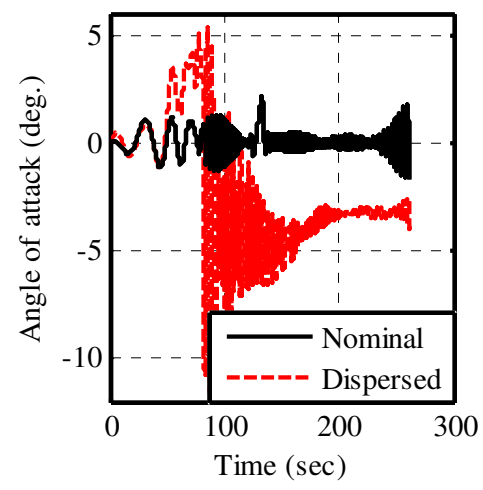

(c) Angle of attack

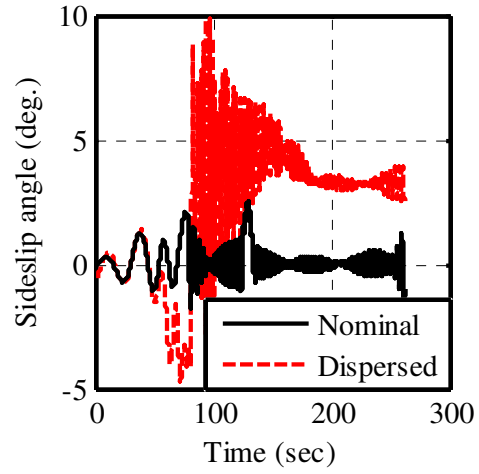

(d) Sideslip angle

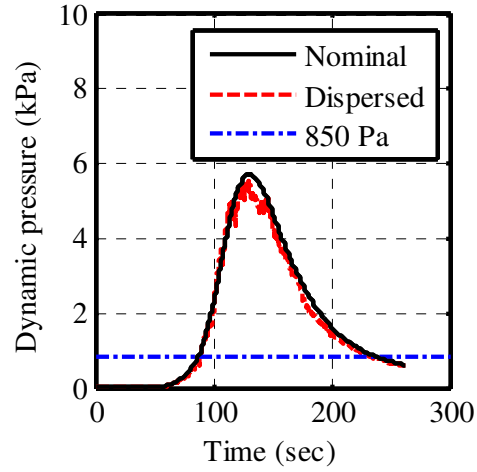

(e) Dynamic pressure

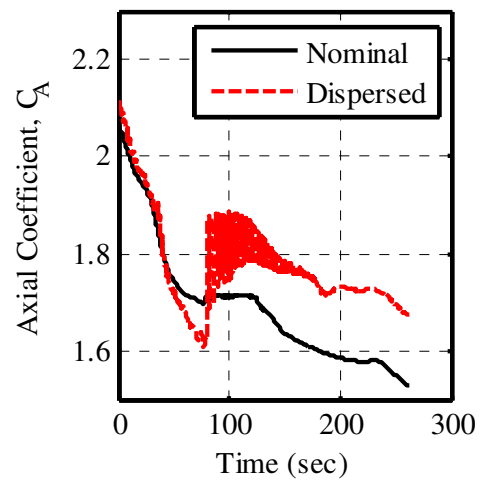

(f) Axial force coefficient

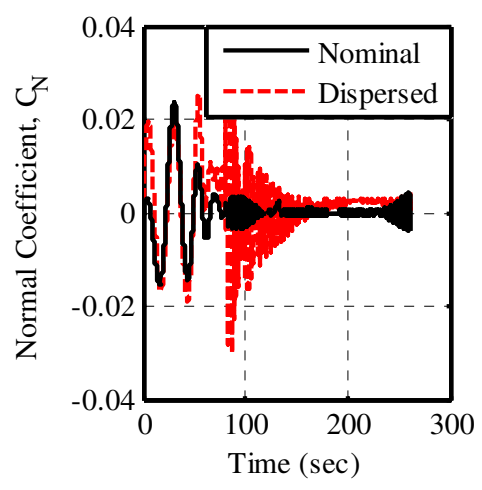

(g) Normal force coefficient

Figure 2. Reference trajectories (nominal and dispersed) used to generate MEDLI-like data.

The POST2 outputs are used to generate IMU measurements, radar altimeter measurements (when the altitude is less than $10 \mathrm{~km}$ ) and MEADS data (when the dynamic pressure is greater than $850 \mathrm{~Pa}$.). Random noise is then applied to the generated data to simulate measurement noise. The uncertainty of the noise that is summarized in Table 2 is based on MEDLI or past Mars EDL instrumentation specifications. Example of the dataset is provided in Figure 3. The radar altimeter data is not shown due to its similarity with the altitude profile shown in Figure 2.

Table 2. Measurement noise uncertainties for the simulated dataset.

\begin{tabular}{llc}
\hline \multicolumn{1}{c}{ Measurement } & \multicolumn{1}{c}{$\mathbf{\pm 3 \sigma}$ uncertainty (normal) } & Sample rate used (Hz) \\
\hline Three-axis sensed acceleration $^{24}$ & $1500 \mu$ g-rms & 4 \\
Three-axis angular rate $^{5}$ & $0.00258 \mathrm{deg} / \mathrm{s}-\mathrm{rms}$ & 4 \\
Radar altimeter altitude $^{24}$ & $0.3 \mathrm{~m}$ & 1 \\
Pressure transducers (MEADS) $^{16}$ & $0.5 \%$ reading/transducer & 4 \\
\hline \hline
\end{tabular}

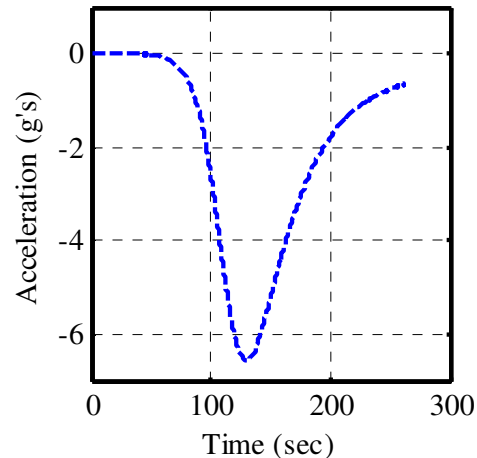

(a) Axial acceleration $\left(\mathrm{A}_{\mathrm{x}, \mathrm{b}}\right)$

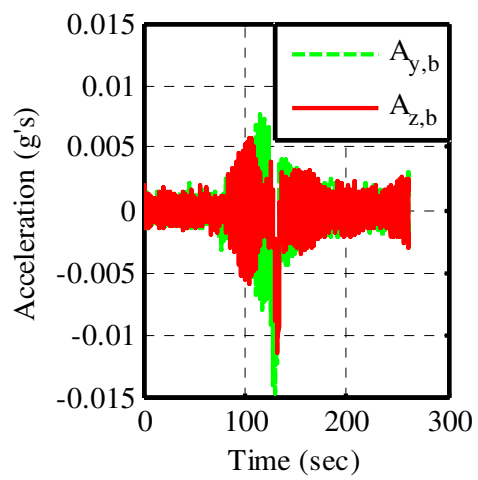

(b) Normal accelerations 


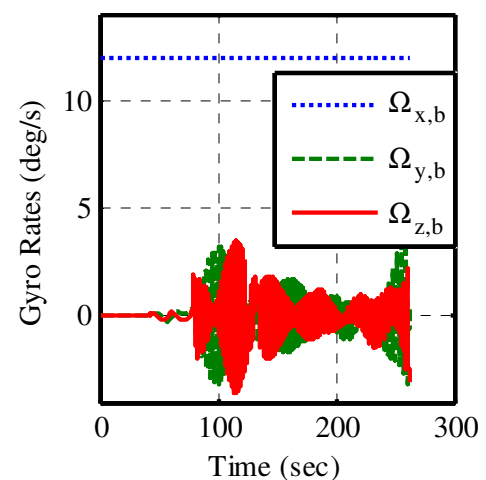

(c) Angular rates

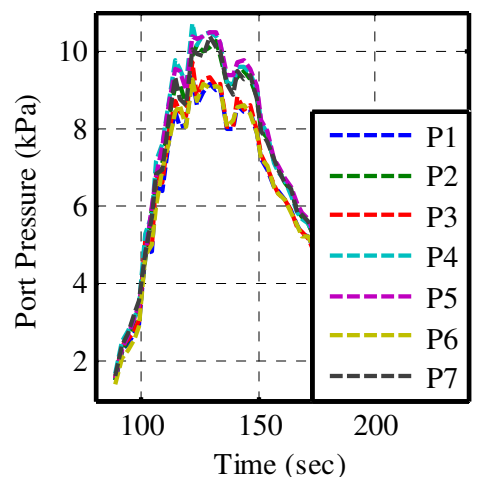

(d) MEADS data

Figure 3. Example of the type of data available for the reconstruction. Radar altimeter data is not shown.

\section{EDL Reconstruction Methodology}

The methodology used for reconstructing Mars EDL vehicle flight parameters (as seen in Figure 4) involves taking EDL sensor measurements and using an estimation method to reconstruct the vehicle trajectory and atmospheric profile. Using the outputs of the estimation method, one can also calculate the aerodynamic coefficients of the vehicle and its uncertainties. Certain formulations of the estimation methods can also allow reconstruction of the sensor calibration data. However, sensor calibration reconstruction is not described in this study.

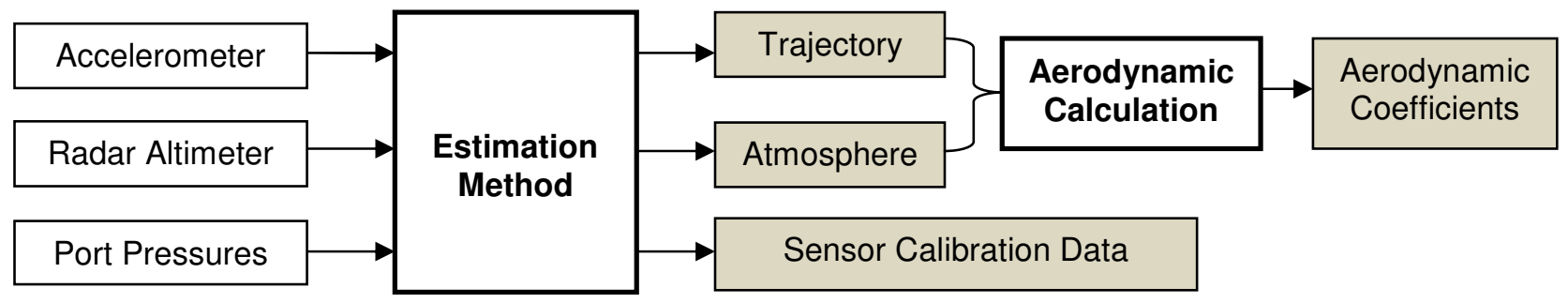

Figure 4. Flow diagram of the overall reconstruction methodology for Mars EDL flight parameters.

The estimation method in Figure 4 will consist of two types of statistical estimators: (1) the Extended Kalman Filter (EKF) and the (2) Unscented Kalman Filter (UKF). The reconstruction starts from an initial condition that is propagated to the time the next measurement is available where the states are updated (Figure 5). Both EKF and UKF are modifications of the original linear Kalman filter that provides the optimal estimate for a linearized system of equations and measurements. ${ }^{25}$ The EDL environment is highly non-linear, so modifications such as the EKF and UKF are needed. Christian et al., ${ }^{24}$ Dutta et al., ${ }^{26,}{ }^{27}$ Karlgaard et al. ${ }^{28}$ and Wells et al ${ }^{29}, 30$ have conducted EDL trajectory and atmosphere reconstruction using EKF. However, either these studies did not test the methodology on a MEDLI-like dataset or the studies did not utilize UKF, which has been shown to improve the parameter estimates and the estimate of the uncertainties compared to linearized estimators, such as EKF. ${ }^{31}$

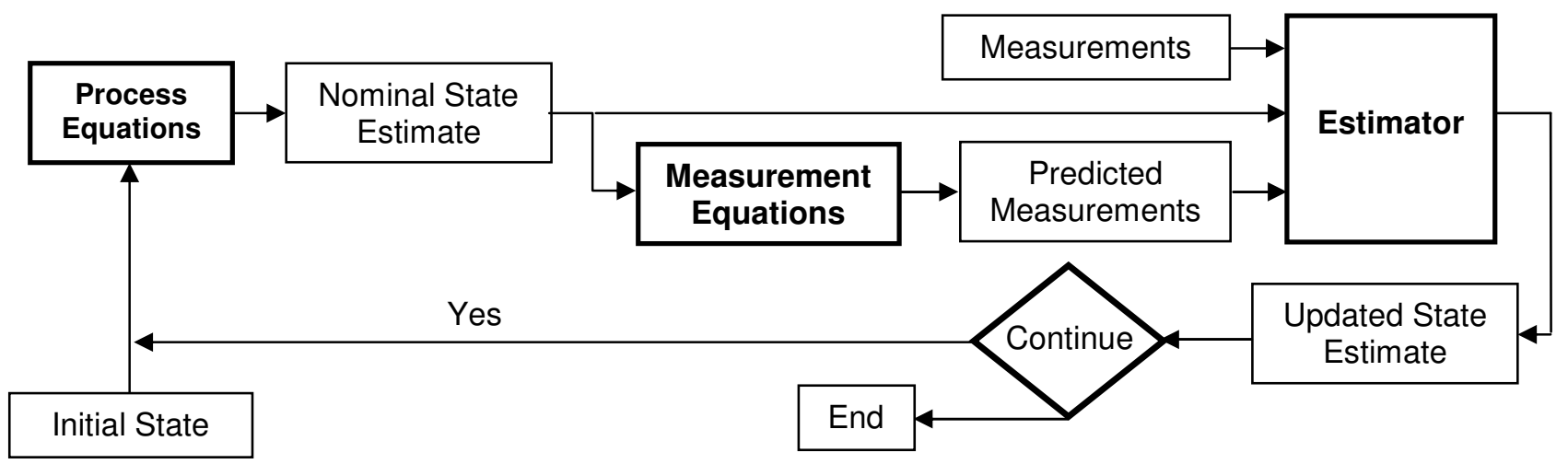

Figure 5. Estimation method flow diagram. 


\section{A. Process Equations}

The estimators need dynamic equations to propagate the estimate of the states in time. For EDL reconstruction, the process equations consist of the equations of motion for the state variables, such as those shown in Eqs. (1). The states consist of the vehicle's position, velocity, attitude, freestream pressure $\left(\mathrm{P}_{\infty}\right)$ and density $\left(\rho_{\infty}\right)$. The position is in terms of planet-centric radius $(\mathrm{r})$, latitude $(\phi)$ and longitude $(\theta)$, while the velocity $(\mathrm{V})$, flight path angle $(\gamma)$ and heading angle $(\psi)$ are defined relative to the planet surface and are based on the vehicle-carried local horizontal frame. ${ }^{31}$ The heading angle is defined in the horizontal plane where due East is $0^{\circ}$ and due North is $90 .^{\circ}$ The attitude states are given in terms of the quaternion $\left(\mathrm{q}_{0}, \mathrm{q}_{1}, \mathrm{q}_{2}, \mathrm{q}_{3}\right)$ that defines the orientation between the vehicle-carried local horizontal frame and the body frame. ${ }^{33,34}$

$$
\begin{gathered}
\dot{r}=V \sin \gamma \\
\dot{\theta}=\frac{V \cos \gamma \cos \psi}{r \cos \phi} \\
\dot{\phi}=\frac{V \cos \gamma \sin \psi}{r} \\
\dot{V}=\frac{F_{T}}{m}-g \sin \gamma+\omega^{2} r \cos \phi(\sin \gamma \cos \phi-\cos \gamma \sin \phi \sin \psi) \\
\dot{\gamma}=\frac{1}{V}\left[\frac{F_{N} \cos V}{m}-g \cos \gamma+\frac{V^{2}}{r} \cos \gamma+2 \omega V \cos \phi \cos \psi+\omega^{2} r \cos \phi(\cos \gamma \cos \phi+\sin \gamma \sin \phi \sin \psi)\right] \\
\dot{\psi}=\frac{1}{V}\left[\frac{F_{N} \sin v}{m \cos \gamma}-\frac{V^{2}}{r} \cos \gamma \cos \psi \tan \phi+2 \omega V(\tan \gamma \cos \phi \sin \psi-\sin \phi)-\frac{\omega^{2} r}{\cos \gamma} \sin \phi \cos \phi \cos \psi\right] \\
\left.\left[\begin{array}{c}
\dot{q}_{0} \\
\dot{q}_{1} \\
\dot{q}_{2} \\
\dot{q}_{3}
\end{array}\right]=\frac{1}{2}\left[\begin{array}{ccc}
-q_{1} & -q_{2} & -q_{3} \\
q_{0} & -q_{3} & q_{2} \\
q_{3} & q_{0} & -q_{1} \\
-q_{2} & q & q_{0}
\end{array}\right]\left[\begin{array}{l}
p \\
q-\frac{1}{r} R_{v b} \\
r
\end{array}\right] \begin{array}{c}
V \cos \gamma \cos \psi \\
-V \cos \gamma \sin \psi \\
\dot{P}_{\infty}=-\rho_{\infty} g V \sin \gamma
\end{array}\right] \\
\dot{\rho}_{\infty}=-\frac{\rho_{\infty}^{2} g V \sin \gamma}{P_{\infty}}
\end{gathered}
$$

The planetary rotation rate is $\omega$. The matrix $R_{\mathrm{vb}}$ defines the rotation from the local horizontal frame to the body frame and is defined in the literature. ${ }^{34} \mathrm{p}, \mathrm{q}, \mathrm{r}$ are the angular rates in the body frame, which come from the on-board gyroscopes, while $g$ is the local gravitational acceleration. $\mathrm{F}_{\mathrm{N}}$ and $\mathrm{F}_{\mathrm{T}}$ represent the normal (lift) and tangential (drag) forces in the body axis. Lift modulation is modeled in the equations using bank angle (v). The freestream pressure and density equations are derived from the hydrostatic equation and the perfect gas law. ${ }^{26,28}$ Isothermal assumption was used to bring the equations to the form shown in Eq. (1h-i). The isothermal assumption may be valid over small changes in the altitude. Since the freestream pressure and density rate equations are used as process equations and are propagated over small time steps, the isothermal assumption is valid.

The process equations used here are not the same equations used internally in POST2. Thus, there is a process uncertainty between how the simulated data is generated and how the estimator predicts the values of the states. In a simulation, one can modify the estimator's process equations to match POST2's dynamics, but in real-life, there are some processes that affect the vehicle that do not have any models. Thus, the difference in the dynamics between POST2 and this estimation methodology is a good test of the unmodeled uncertainties expected in the actual data.

\section{B. Measurement Equations}

Measurement equations are used by the statistical estimator to predict the measurement value based on the current estimate of the state. EKF and UKF assume that the measurement has a Gaussian error distribution and the expectation of the error is zero. The EKF approximates the measurement equation by linearizing about a point (the nominal estimate of the state). A measurement sensitivity (Jacobian) matrix is involved in the linearization and this matrix is computationally intensive. This Jacobian matrix is not necessary for the UKF.

When using the EKF, the measurement sensitivity equations have to be developed for every measurement type included in the estimators. Christian et al., ${ }^{24}$ Karlgaard et al. ${ }^{28}$ and Jaswinski et al. ${ }^{35}$ provide detailed expressions for the measurement sensitivity equations pertaining to accelerometer and radar altimeter measurements. For MEDLI data, the measurement equation has to give predicted static pressure value at a specific transducer. The static 
pressure value is a function of three parameters: total angle of attack $\left(\alpha_{t}\right)$, freestream Mach number $\left(\mathrm{M}_{\infty}\right)$ and the orientation of the transducer on the aeroshell. ${ }^{26}$ During the hypersonic EDL phase, the velocity of the vehicle is large with respect to the wind velocity. So the planet-relative velocity can be used to calculate the angle of attack and angle of sideslip (Eqs. (2)). The relative velocity to the wind should be used for more precision. Wind speed could be included in the state vector, but an equation of motion for the wind speed must then be included. For simplicity, the winds are not modeled in this study. The two orientation angles can then be combined into a total angle of attack (also shown in Eqs. (2)). Note that $\mathrm{u}, \mathrm{v}$ and $\mathrm{w}$ are velocity components in the body axis.

$$
\begin{gathered}
\alpha=\tan ^{-1}(w / u) \\
\beta=\sin ^{-1}(v / V) \\
\alpha_{t}=\cos ^{-1}(\cos \alpha \cos \beta)
\end{gathered}
$$

The velocity magnitude can be used to calculate the local Mach number and the speed of sound needed for the Mach number calculation is a function of the freestream pressure and density that are part of the state vector. The locations of the pressure measurement orifices are also known. The pressure coefficient $\left(\mathrm{C}_{\mathrm{p}}\right)$ at each orifice can then be found from tables created from the vehicle aerodynamic database. An example of such a table is given in Ref. 26. After the pressure coefficient is found, the pressure at each surface location can be predicted using the vehicle velocity and density. Measurement sensitivity expressions for pressure data are developed by numerical differentiation due to a lack of an analytical expression relating the state vector with the predicted port pressure. Numerical ill-conditioning can arise based on the tolerance value used to perturb the pressure prediction equations.

\section{Statistical Estimators}

\section{Extended Kalman Filter}

An extended Kalman filter is a modification of the original Kalman filter to express the nonlinearity in the system dynamics that is lost in the linearization needed for the original Kalman filter. Instead of estimating perturbations about a nominal trajectory, the EKF uses the nonlinear equations of state to propagate the state vector. ${ }^{36}$ The algorithm for this filter can be summarized as follows ${ }^{37}$

1. Initialize the state vector and the state covariance matrix at time $\mathrm{t}_{\mathrm{k}-1}=\mathrm{t}_{0}$ and let $\mathrm{k}=1$, where $\mathrm{k}$ is an index of the epoch when a measurement is first available.

2. Read in the measurement at time $t_{\mathrm{k}}$.

3. Calculate a nominal state at $\mathrm{t}_{\mathrm{k}}\left(\hat{x}_{k}^{-}\right)$by integrating the non-linear equations of motions (Eqs. (1)) with $\hat{x}_{k-1}^{+}$as the initial condition.

4. Calculate the nominal state covariance matrix $\left(\hat{P}_{k}^{-}\right)$by integrating the Riccati equations (Eq. (3a)).

5. Calculate the measurement residual vector $\left(\mathrm{y}_{\mathrm{k}}\right)$, the measurement sensitivity matrix $\left(\mathrm{H}_{\mathrm{k}}\right)$, and the Kalman gain $\left(\mathrm{K}_{\mathrm{k}}\right)$ using the nominal state and state covariance (Eq. (3b)).

6. Calculate the best estimate of the state $\left(\hat{x}_{k}^{+}\right)$and state covariance $\left(\hat{P}_{k}^{+}\right)$using Eqs. (3c) and (3d).

7. Increment counter $\mathrm{k}$ and go back to step 2 until measurements at all times have been processed.

$$
\begin{gathered}
\dot{P}=A P+P A^{T}+B Q B^{T} \\
K_{k}=\hat{P}_{k}^{-} H_{k}^{T}\left(H_{k} \hat{P}_{k}^{-} H_{k}^{T}+R_{k}\right)^{-1} \\
\hat{x}_{k}^{+}=\hat{x}_{k}^{-}+K_{k}\left(y_{k}-h\left(\hat{x}_{k}^{-}\right)\right) \\
\hat{P}_{k}^{+}=\left(I-K_{k} H_{k}\right) \hat{P}_{k}^{-}\left(I-K_{k} H_{k}\right)^{T}+K_{k} R_{k} K_{k}^{T}
\end{gathered}
$$

The measurement covariance matrix $(\mathrm{R})$ is defined at time $\mathrm{k}$ and information from Table 2 is used for this matrix. Q is the process noise covariance, A is the Jacobian of the equations of motion with respect to the state vector and B is the Jacobian of the equations of state with respect to the state noise vector. I is the identity matrix.

\section{Unscented Kalman Filter}

Instead of using a linearized approximation to update the state and covariance matrix, the UKF is based on the idea that a transformation of a probability distribution can be approximated using an arbitrary nonlinear function. ${ }^{31}$ Just like the EKF, the UKF assumes that the state variables are Gaussian distributions in which the state estimates are the means and the state uncertainties are the standard deviations of the distributions. The UKF propagates a set 
of specially chosen state vectors called sigma points to characterize the transformation of the state probability distribution. The definition of the sigma points and how they are propagated in time are shown in Eqs. (4), ${ }^{31,38}$ where $\mathrm{n}$ is the number of elements in the state space. $\alpha, \beta$ and $\kappa$ are user defined tuning constants used to modify the performance of the filter. $\kappa$ is usually 0 or $3-\mathrm{n}, \beta=2$ when $\mathrm{x}$ is Gaussian, $\alpha \sim 1 \mathrm{x} 10^{-3}$ and $\lambda \sim \alpha^{2}(\mathrm{n}+\kappa)-\mathrm{n} .{ }^{31,39} \mathrm{~W}$ is the weights for the $(2 n+1)$ sigma points and $g$ is the nonlinear transformation. Note that superscript " $b$ " indicates the state and covariance before the transformation and the superscript " $a$ " indicates values after the transformation.

$$
\begin{gathered}
x^{(0)}=\bar{x} \\
x^{(i)}=\bar{x}+\tilde{x}^{(i)} \quad i=1, \ldots . . n \\
\tilde{x}^{(i)}=(\sqrt{(n+\lambda) P})_{i}^{T} \quad \tilde{x}^{(n+i)}=-(\sqrt{(n+\lambda) P})_{i}^{T} i=1, \ldots . n \\
W_{\text {mean }}{ }^{(0)}=\frac{\lambda}{n+\lambda} \\
W_{\text {covariance }}{ }^{(0)}=W_{\text {mean }}{ }^{(0)}+1-\alpha^{2}+\beta \\
W^{(i)}=\frac{1}{2(n+\lambda)} \quad i=1, \ldots n \\
x^{a(i)}=g\left(x^{b(i)}\right) \\
\bar{x}^{a}=\sum_{i=0}^{2 n} W^{(i)} x^{a(i)} \\
P^{a}=\sum_{i=0}^{2 n} W^{(i)}\left(x^{a(i)}-\bar{x}^{a}\right)\left(x^{a(i)}-\bar{x}^{a}\right)^{T}+Q_{k-1}
\end{gathered}
$$

A new set of sigma points need to be calculated from $\hat{x}_{k}^{-}$for the measurement equations. The predicted measurement for each sigma point $\left(\hat{y}_{k}^{(i)}\right)$ and the estimate of the mean value for the predicted measurement $\left(\hat{y}_{k}\right)$ can be calculated using Eqs. (5). The covariance of the predicted measurement $\left(\mathrm{P}_{\mathrm{y}}\right)$ and the cross covariance between the estimated state and measurement $\left(\mathrm{P}_{\mathrm{xy}}\right)$ are used for the Kalman gain and state update steps. ${ }^{31,38,39}$

$$
\begin{gathered}
P_{y}=\sum_{i=0}^{2 n} W^{(i)}\left(\hat{y}_{k}^{(i)}-\hat{y}_{k}\right)\left(\hat{y}_{k}^{(i)}-\hat{y}_{k}\right)^{T}+R_{k} \\
P_{x y}=\sum_{i=0}^{2 n} W^{(i)}\left(\hat{x}_{k}^{(i)}-\hat{x}_{k}\right)\left(\hat{y}_{k}^{(i)}-\hat{y}_{k}\right)^{T}+R_{k} \\
K_{k}=P_{x y} P_{y}^{-1} \\
\hat{x}_{k}^{+}=\hat{x}_{k}^{-}+K_{k}\left(y_{k}-\hat{y}_{k}\right) \\
\hat{P}_{k}^{+}=\hat{P}_{k}^{-}-K_{k} P_{y} K_{k}^{T}
\end{gathered}
$$

\section{Statistical Smoothing}

The reconstruction can start from the atmospheric entry (forward pass) or a projected landing location (backwards pass). The forward pass starts its estimate from an initial state and covariance that is found independent of the trajectory reconstruction process. The reconstruction is conducted in a chronological manner. The backwards pass has the advantage of starting at a smaller uncertainty value as it begins from the end of the forward estimate. The forward (f) and backward (b) pass estimates can be combined using the Fraser-Potter smoothing solution, ${ }^{40}$ which is shown in Eqs. 6. An advantage of combining both the forward and backward estimates is to find an optimal estimate of the trajectory. The forward pass estimate at time $\mathrm{k}$ uses the measurement data from entry to $\mathrm{k}$, while the backward pass uses the measurement data from landing time to $\mathrm{k}$. The combined smoothed estimate can use measurement data at all times to create the estimate at $\mathrm{k}$.

$$
\begin{gathered}
\hat{P}_{k}=\left[\hat{P}_{f, k}^{-1}+\hat{P}_{b, k}^{-1}\right]^{-1} \\
\hat{x}_{k}=\hat{P}_{k}\left[\hat{P}_{f, k}^{-1} \hat{x}_{f, k}+\hat{P}_{b, k}^{-1} \hat{x}_{b, k}\right]^{-1}
\end{gathered}
$$

\section{Aerodynamic Reconstruction}

The aerodynamic coefficients of the vehicle can be constructed after trajectory and atmospheric states have been estimated. As seen in Eq. (7), aerodynamic force coefficients (in this case the axial force coefficient $\left(\mathrm{C}_{\mathrm{A}}\right)$ ) can be reconstructed from the acceleration measurements $\left(\mathrm{a}_{\mathrm{x}}\right)$ and the estimated freestream density and velocity values. 
With the estimation process complete, uncertainty values are known for the accelerations and the freestream properties. In this manner, the aerodynamic and atmospheric uncertainties can be separated. The uncertainty in the estimate of the aerodynamic force coefficients can be calculated by applying the chain rule to Eq. (7) and using the already calculated uncertainties of the estimator's state vector.

$$
C_{A}=\frac{m a_{x}}{0.5 \rho_{\infty} V_{\infty}^{2} A}
$$

\section{Nominal Dataset Reconstruction}

The reconstruction process for both datasets (nominal and dispersed) begins with the same initial conditions and initial covariance values. The initial uncertainties in the state variables are listed in Table 3 and are based on the initial conditions at entry interface from recent Mars missions. The initial state covariance matrix is calculated from these values assuming that there is no correlation between the state variables. The process noise covariance is calculated using the uncertainty information given in Table 4. Process noise improves the estimator's ability to reconstruct parameters from noisy data and model uncertainties in the process equations. ${ }^{35-38}$ The process noise is high for freestream pressure and density as these two states are propagated using the dynamic equations based on the isothermal assumption of the hydrostatic equation and the perfect gas law. The process noise is high to demonstrate the relatively high uncertainty in the process equations so that the estimator is biased towards the more certain measurements from the accelerometer and MEDLI-like pressure transducers.

Table 3. Initial state uncertainties used for the reconstruction process.

\begin{tabular}{|c|c|}
\hline State & $\begin{array}{c} \pm 3 \sigma \text { uncertainty } \\
\text { (normal) }\end{array}$ \\
\hline Radius (planet-centric) ${ }^{24}$ & $5100 \mathrm{~m}$ \\
\hline Latitude (planet-centric) ${ }^{24}$ & $0.12 \mathrm{deg}$. \\
\hline Longitude $^{24}$ & 0.03 deg. \\
\hline Velocity (relative) ${ }^{5}$ & $0.3 \mathrm{~m} / \mathrm{s}$ \\
\hline Flight path angle (relative) ${ }^{24}$ & $0.06 \mathrm{deg}$. \\
\hline Heading angle (relative) ${ }^{24}$ & $0.06 \mathrm{deg}$. \\
\hline $\begin{array}{l}\text { Euler angles } \\
\text { (related to the quaternion) }\end{array}$ & 0.03 deg./angle \\
\hline Freestream pressure & $10 * \mathrm{P}_{\infty .0}$ \\
\hline Freestream density & $10^{*} \rho_{\infty .0}$ \\
\hline
\end{tabular}

Table 4. Process noise uncertainties used for the reconstruction process.

\begin{tabular}{ll}
\hline \hline \multicolumn{1}{c}{ State } & \multicolumn{1}{c}{$\begin{array}{c}\mathbf{1} \boldsymbol{3} \boldsymbol{\sigma} \text { uncertainty } \\
\text { (normal) }\end{array}$} \\
\hline Radius (planet-centric) & $0.003 \mathrm{~m}$ \\
Latitude (planet-centric) & $150 \mu \mathrm{deg}$. \\
Longitude & $150 \mu \mathrm{deg}$. \\
Velocity (relative) & $0.003 \mathrm{~m} / \mathrm{s}$ \\
Flight path angle (relative) & $0.015 \mathrm{deg}$. \\
Heading angle (relative) & $0.015 \mathrm{deg}$. \\
Quaternion & Based on angular rate \\
Freestream pressure & measurement noise \\
Freestream density & $0.3 * \mathrm{P}_{\infty}$ \\
\hline \hline
\end{tabular}

The reconstruction results of the MEDLI-related parameters using the nominal simulated dataset are shown in Figure 6. The deviations of the reconstructed parameters are from the true values shown in Figure 2.

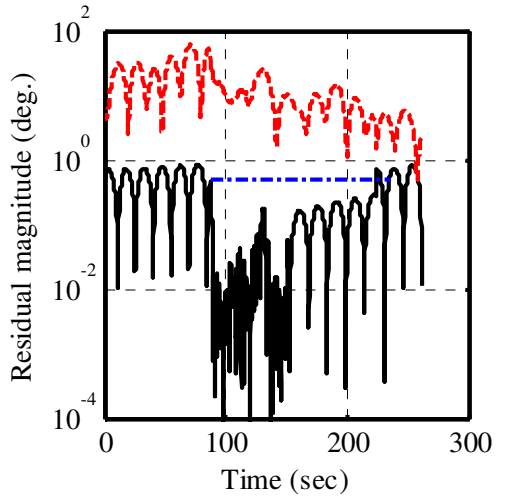

(a) Angle of attack (EKF)

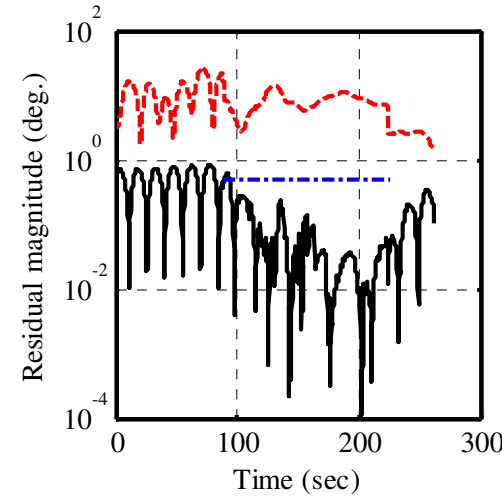

(b) Angle of attack (UKF)

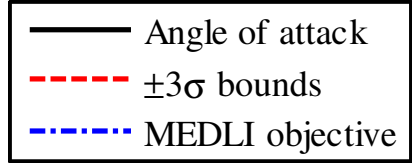




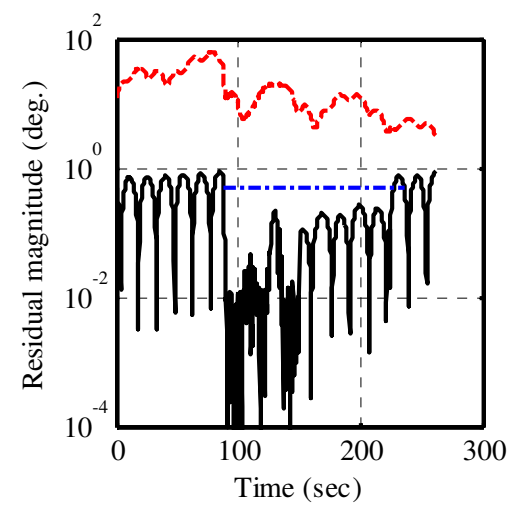

(c) Sideslip angle (EKF)

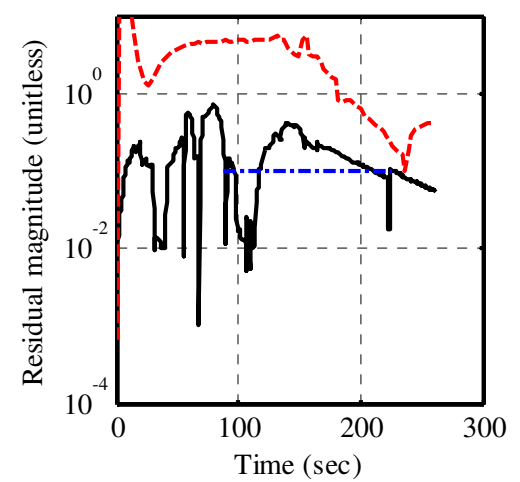

(e) Mach number (EKF)

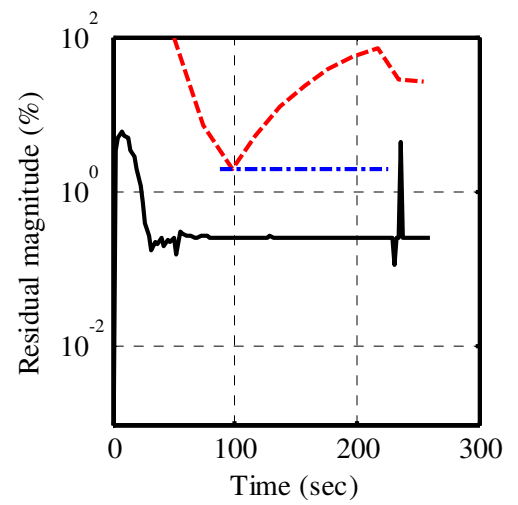

(g) Dynamic pressure (EKF)

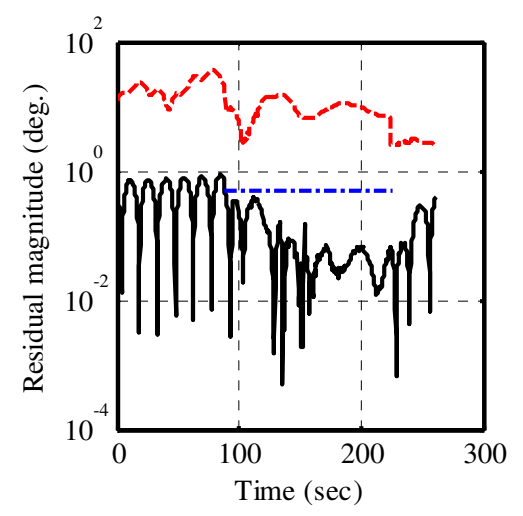

(d) Sideslip angle (UKF)

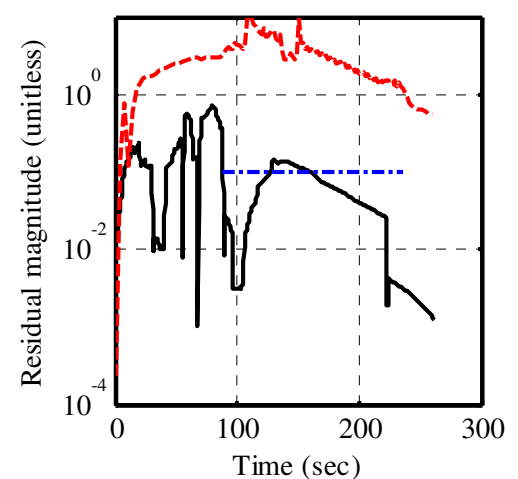

(f) Mach number (UKF)

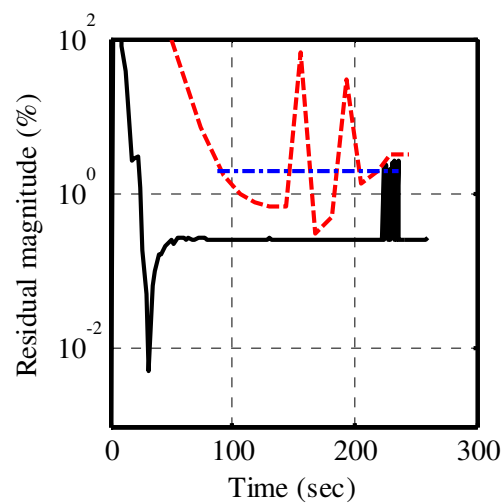

(h) Dynamic pressure (UKF)
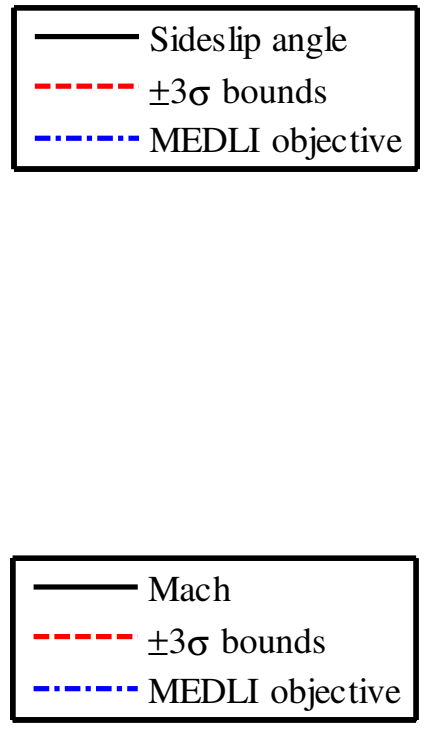

Figure 6. Reconstructed MEDLI-related parameters' deviation from the truth using the nominal dataset.

For the most part, it appears that the trajectory and atmospheric parameters are reconstructed close to the MEDLI objectives, especially in the region where MEDLI data is available. The Mach number estimate does stray from the MEDLI objective bounds for both the EKF and UKF, but the UKF estimate's residual is lower than EKF estimate's residual during the time MEDLI data is available. Note that the MEDLI science goals are at \pm 0.1 , while the science goals for the other parameters are less stringent (e.g. $\alpha$ reconstruction goal is \pm 0.5 deg.). The Mach number value is also dependent on the calculated speed of sound, which in turn relies on the estimated freestream pressure and density. These parameters are estimated using the MEDLI data that peak around the time period (Figure 3d) when the Mach number is outside the MEDLI objective bounds. The MEDLI data have a simulated noise that is a percentage of the nominal measurement and the data are noisiest in this region. This nonlinearity manifests itself in the reconstructed freestream atmospheric parameters and Mach number. Another source for the speed of sound profile, e.g. using MISP data to reconstruct the freestream temperature, may reduce this deviation. MISP data is 
currently not planned to be used for this purpose, but future work could involve doing a simultaneous trajectory, atmosphere, aerodynamic and aerothermodynamic reconstruction where MEADS and MISP data are both used.

The estimated uncertainties presented are the $99.7 \%( \pm 3 \sigma)$ confidence interval since the states are assumed to be Gaussian distributions and the residual between the estimated states and the truth fall within these confidence bands. In practice, Monte Carlo analysis performed for a given trajectory and the possible deviations in trajectories will be compared with the estimated uncertainties. However, for this study there is no Monte Carlo data available to compare with the estimated uncertainties. Such analysis is planned for future work.

Additionally, since the states are assumed to be Gaussian distributions, the tails of the distributions theoretically stretch from positive infinity to negative infinity. Of course, in real life the actual states cannot be distributed in such a manner. The estimate states agree well with the truth, but surprisingly the estimated uncertainties for some of the states (like dynamic pressure) seem unrealistic and could simply be artifacts of a Gaussian estimator. More work needs to be done to refine the uncertainty estimates using tuning parameters.

Due to a lack of true uncertainty values, it is hard to decide if UKF does a better job of estimating uncertainties than the EKF, as predicted in the literature. However, the estimate's residual magnitude for the UKF is definitely lower for some variables (such as Mach number) than EKF's estimate residual magnitude. Thus, the UKF demonstrates a better ability in state estimation than the EKF as the literature predicts.

Trajectory parameters, such as planet-centric radius and planet-relative velocity are also reconstructed in the estimation process. The percent deviation from the truth for the trajectory parameters can be seen in Figure 7. Both the EKF and the UKF do a good job of estimating the radius (within $0.2 \%$ ) and the velocity, although the UKF estimate's residual magnitude is lower than the EKF estimate's residual. Note that the radius and its uncertainty estimation improve significantly with the introduction of radar altimeter measurement around 220 seconds.

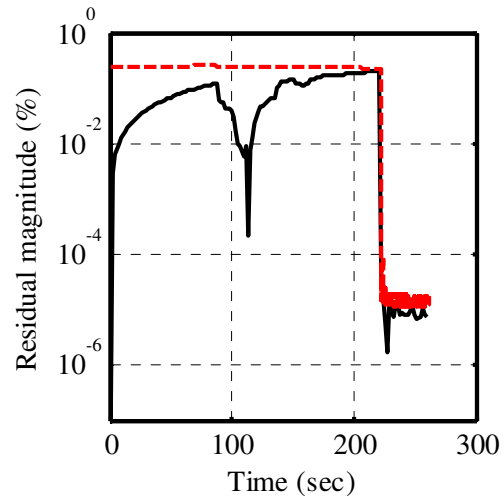

(a) Radius (EKF)

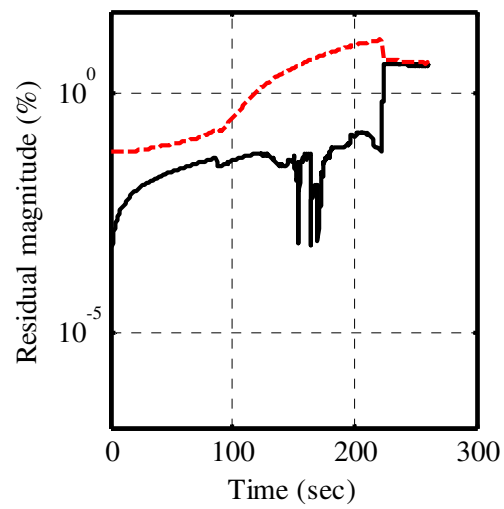

(c) Velocity (EKF)

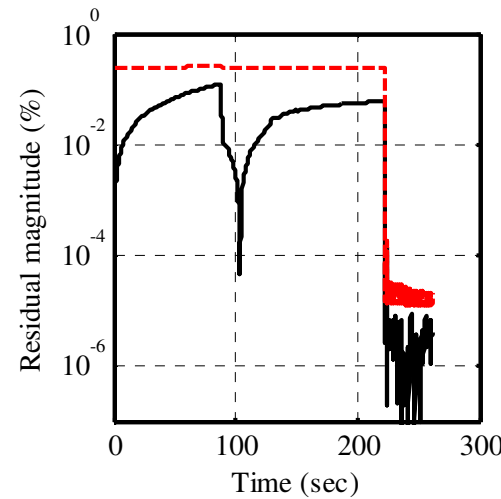

(b) Radius (UKF)

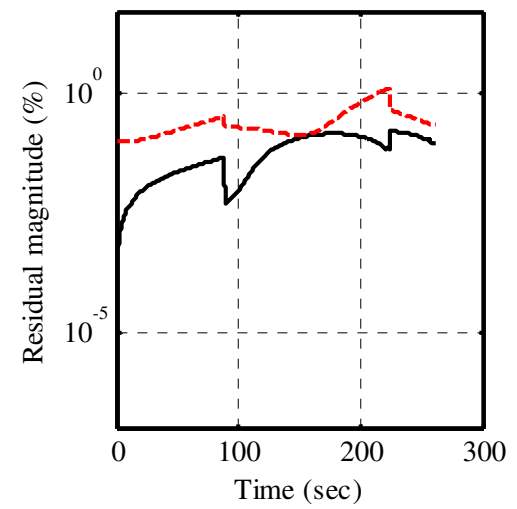

(d) Velocity (UKF)
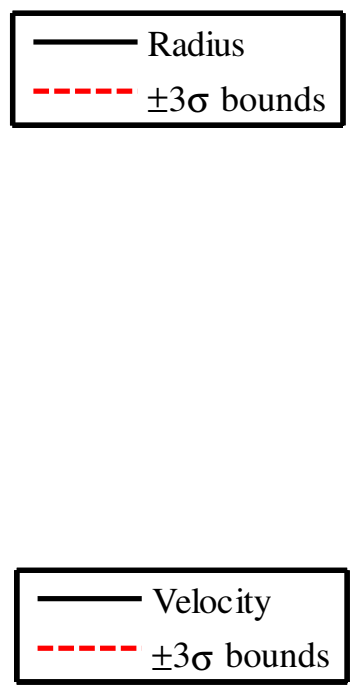

Figure 7. Deviation of the reconstructed trajectory from the truth using the nominal simulated dataset.

Figure 8 shows the reconstructed aerodynamic force coefficients for the time span that MEDLI data was simulated since the freestream pressure and density are directly observable for only this time period. The reconstructed axial force coefficient appears to be very close to the truth, as the coefficient's deviation for both the EKF and UKF estimate lies within \pm 0.025 for most of the time period of interest. However, the true normal force

12

American Institute of Aeronautics and Astronautics 
coefficient $\left(\mathrm{C}_{\mathrm{N}}\right)$ has a very small value (Figure $2 \mathrm{~g}$ ), which raises a numerical issue as both estimators do not estimate $\mathrm{C}_{\mathrm{N}}$ to the same percentage accuracy as they estimate $\mathrm{C}_{\mathrm{A}}$. Although the residuals for $\mathrm{C}_{\mathrm{A}}$ and $\mathrm{C}_{\mathrm{N}}$ are of the same order of magnitude, the residual for $C_{N}$ is only one order of magnitude lower than its nominal value. The estimates for $C_{A}$ and $\mathrm{C}_{\mathrm{N}}$ are pretty good (demonstrated by their low residuals), but the $\mathrm{C}_{\mathrm{A}}$ estimate is better by percentage accuracy.

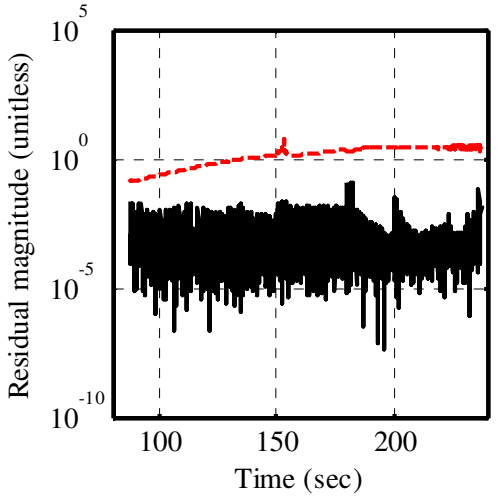

(a) Axial force coefficient residual (EKF)

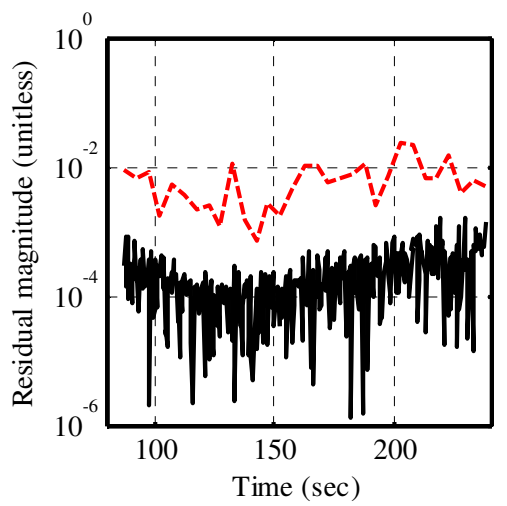

(c) Normal force coefficient residual $(\mathrm{EKF})$

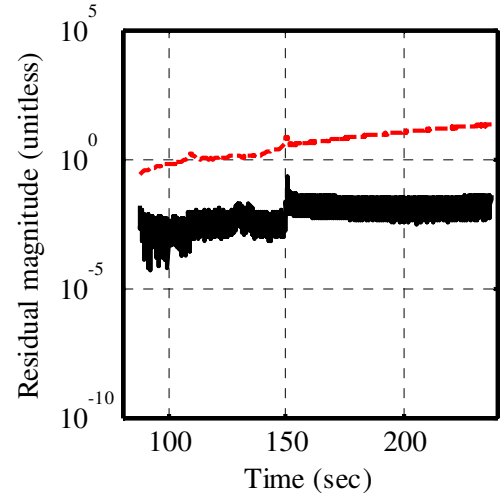

(b) Axial force coefficient residual (UKF)

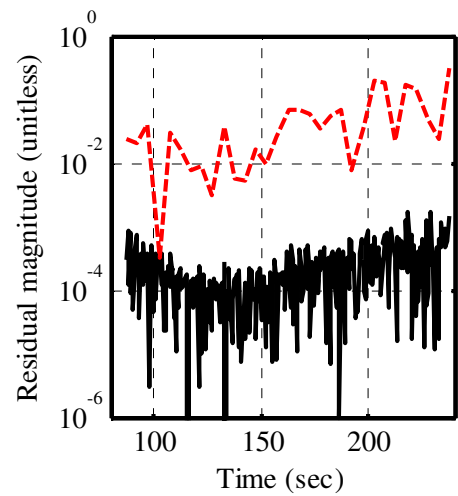

(d) Normal force coefficient residual (UKF)
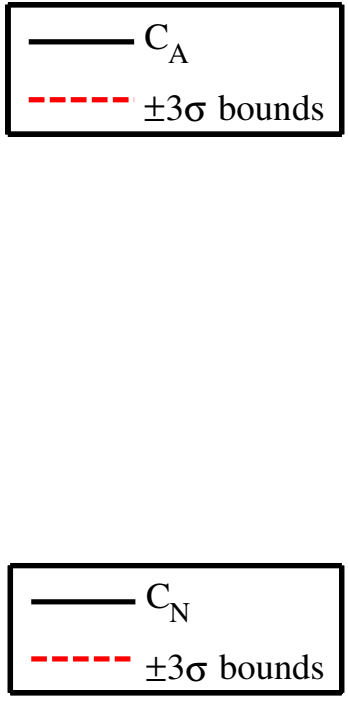

Figure 8. Comparison of the estimated aerodynamic parameters from the truth using the nominal dataset.

The $\pm 3 \sigma$ bounds show that the $C_{A}$ and $C_{N}$ estimate's uncertainty is approximately \pm 1 and $\pm 1 \times 10^{-2}$. For nominal $\mathrm{C}_{\mathrm{A}}$ values of about 1 , an error band of \pm 1 may be pretty good, but for nominal $\mathrm{C}_{\mathrm{N}}$ values that are of the order $1 \times 10^{-3}$, an error band of $\pm 1 \times 10^{-2}$ is very large. This reinforces the need for further research with tuning parameters to improve upon the uncertainty estimation, especially for parameters with nominal values close to zero, such as $\mathrm{C}_{\mathrm{N}}$.

\section{Dispersed Dataset Reconstruction}

The estimate of the MEDLI-related parameters using the dispersed simulated dataset is shown in Figure 9.

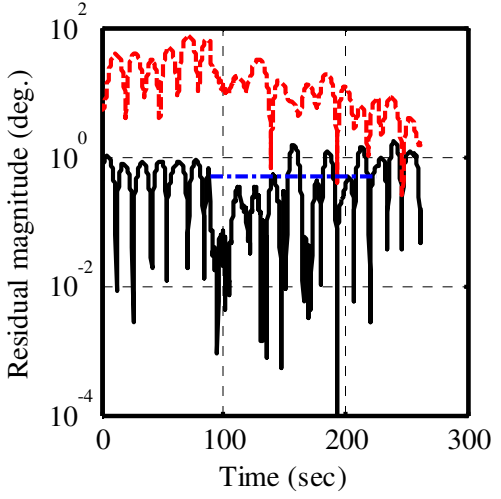

(a) Angle of attack (EKF)

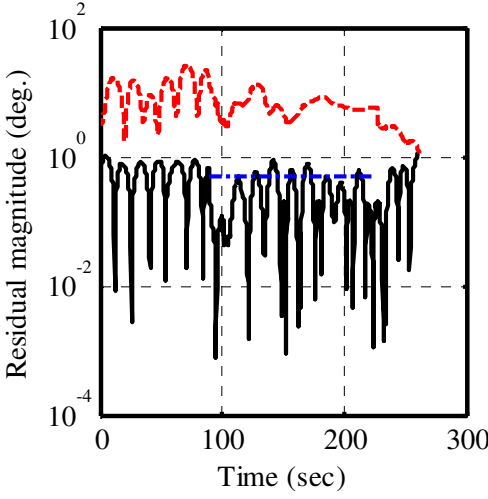

(b) Angle of attack (UKF)

13

American Institute of Aeronautics and Astronautics 


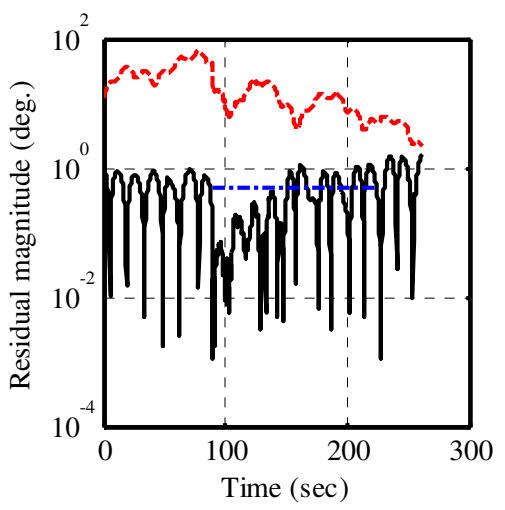

(c) Sideslip angle (EKF)

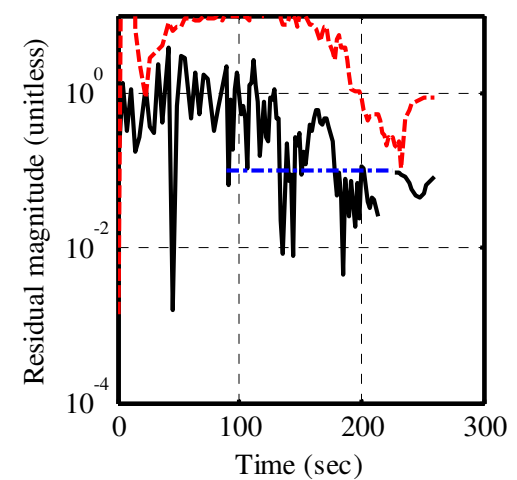

(e) Mach number (EKF)

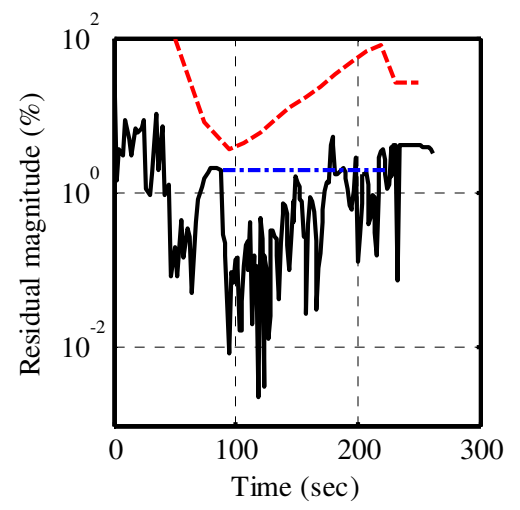

(g) Dynamic pressure (EKF)

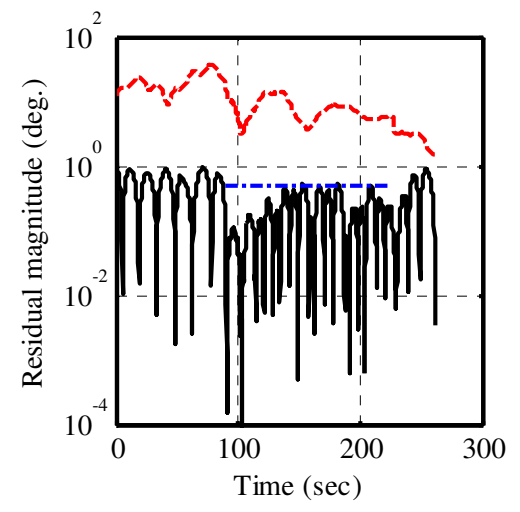

(d) Sideslip angle (UKF)

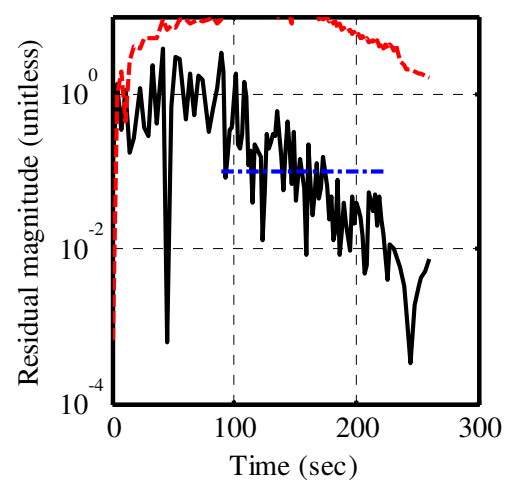

(f) Mach number (UKF)

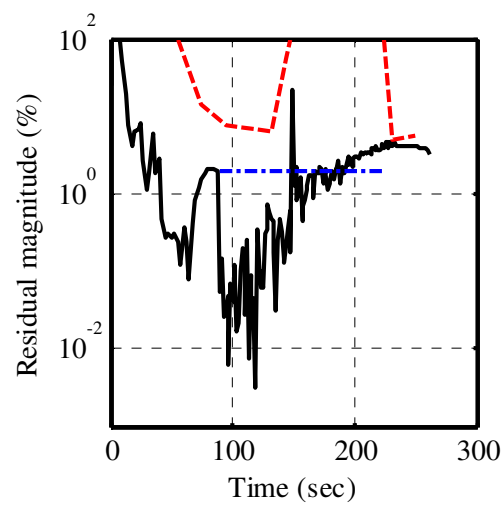

(h) Dynamic pressure (UKF)
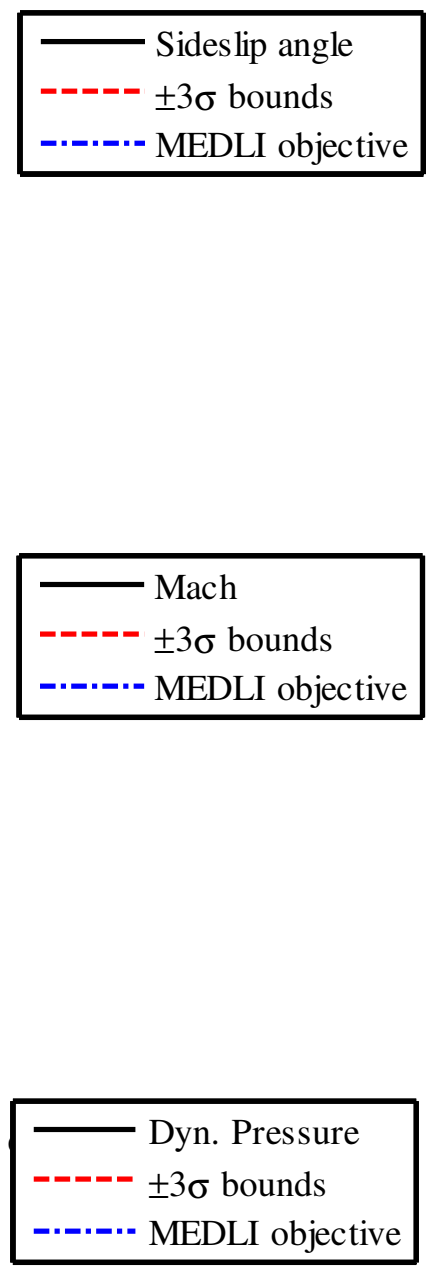

Figure 9. Reconstructed MEDLI-related parameters' deviation from the truth using the dispersed dataset.

Compared to the reconstructed parameters from the nominal dataset, the deviations of the estimates from the truth appear to be larger and noisier, which could be reflecting the perturbations in aerodynamics and atmosphere modeled in this trajectory. Once again, the estimated angle of attack, sideslip angle and dynamic pressure meet the MEDLI science objectives for the most part, while the estimated Mach number (Figure 9e-f) strays from the MEDLI objective. The rationale for this deviation is similar to what was previously stated. Note that in this case even the true Mach number profile shows significant variation in this region (Figure 2b). This highly non-linear behavior affects the estimation performance of this parameter. Moreover, the tuning parameters and process noise for the nominal and dispersed datasets were kept constant in this study for the ease of comparison. However, modifying the tuning parameters and process noise (e.g. increasing the process noise to reflect the perturbations in the trajectory) will most likely improve the estimation of parameters using the dispersed dataset.

The percent deviation from the truth for the trajectory parameters can be seen in Figure 10. Once again, both the EKF and the UKF do a good job of estimating the radius and the velocity, although the UKF estimate's residual magnitude is lower than the EKF estimate's residual for radius. 


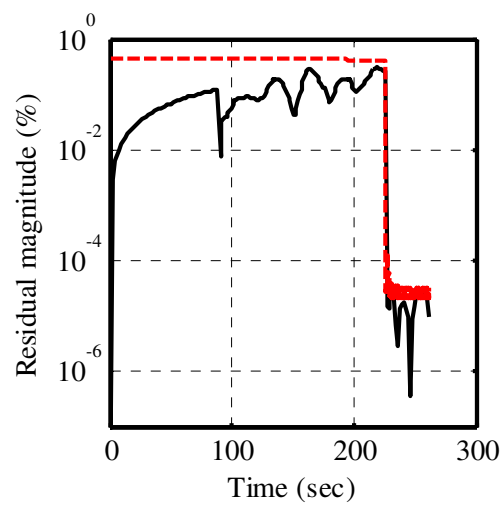

(a) Radius (EKF)

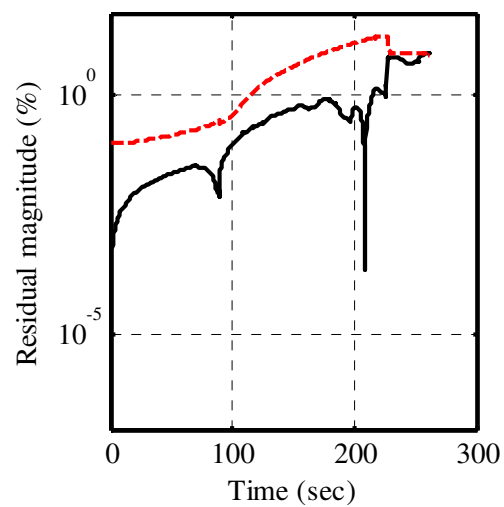

(c) Velocity (EKF)

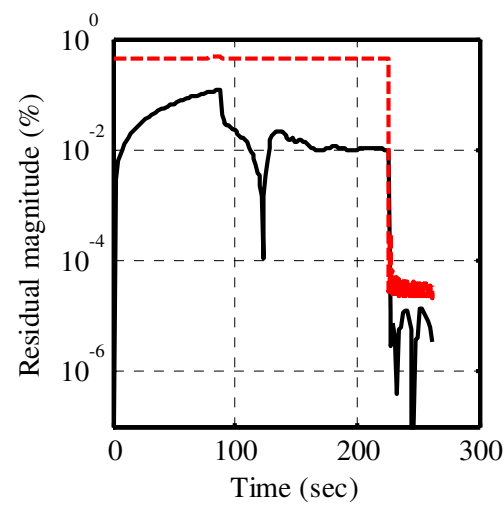

(b) Radius (UKF)

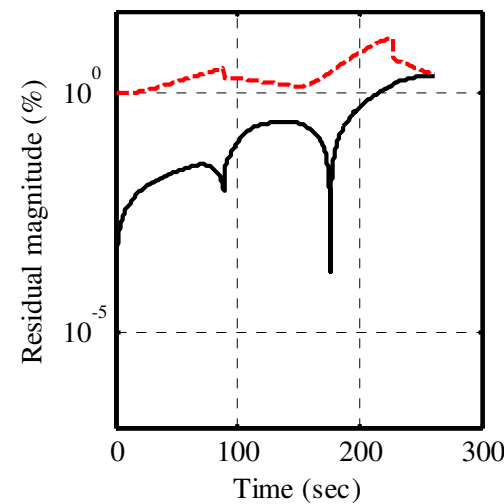

(d) Velocity (UKF)

Figure 10. Deviation of the reconstructed trajectory from the truth using the dispersed simulated dataset.

The reconstructed aerodynamic force coefficients for the dispersed dataset are shown in Figure 11. The small values for the normal coefficient again raise the same numerical issues earlier discussed.

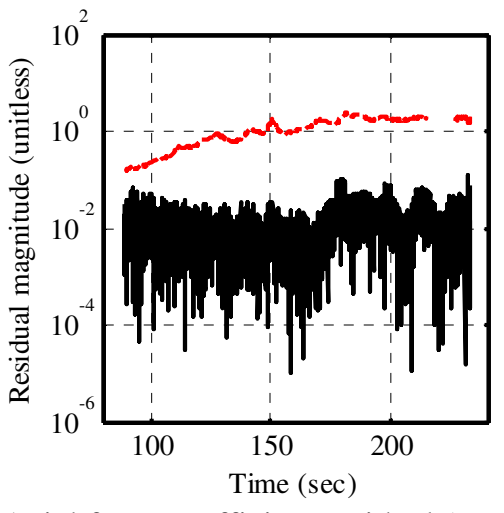

(a) Axial force coefficient residual (EKF)

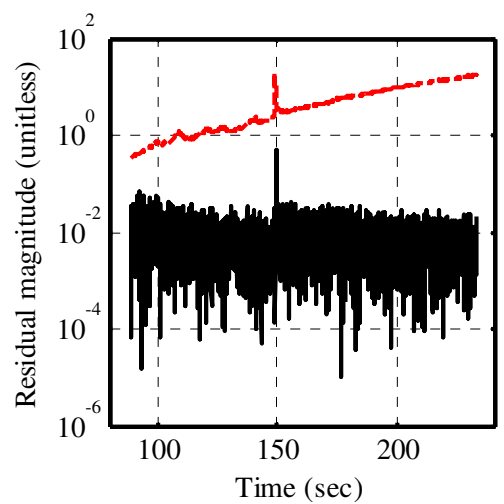

(b) Axial force coefficient residual (UKF)

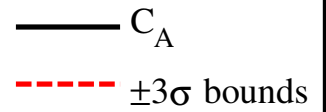




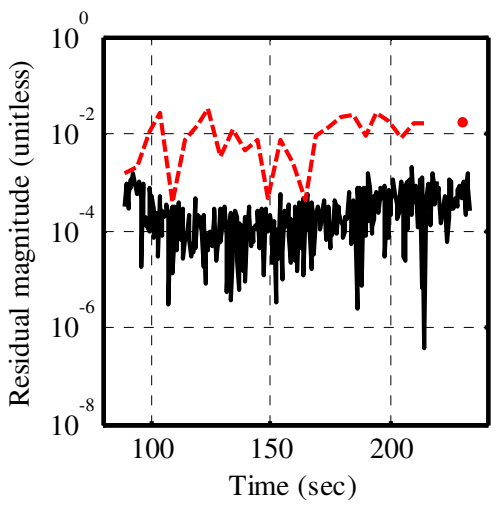

(c) Normal force coefficient residual (EKF)

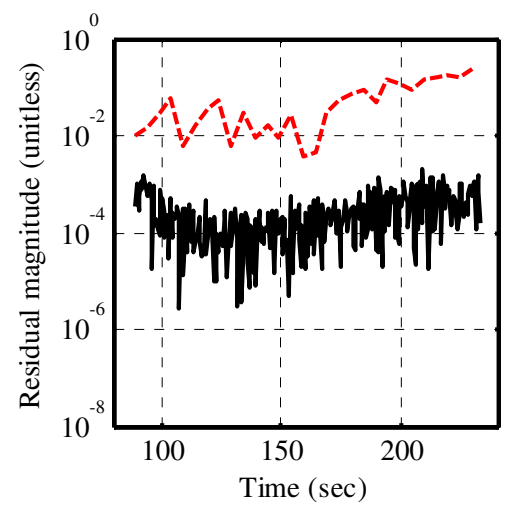

(d) Normal force coefficient residual (UKF)

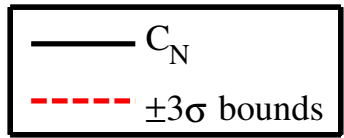

Figure 11. Comparison of the estimated aerodynamic parameters from the truth using the dispersed dataset.

\section{Comparison between the Estimators}

It appears that the EKF and UKF have generally similar performance. However, there are some situations where the UKF outperforms the EKF. Looking at the reconstruction of the angle of attack and sideslip angle (Figure 6a-d and Figure 9a-d), one can see that the UKF reconstruction largely stays within the MEDLI science objective goals while the EKF has a large deviation near the end of the time period that the MEDLI dataset is available. This type of behavior is expected from the EKF as the errors due to linearization lead to divergence from the truth when the errors propagate over a long time period. The UKF is able to avoid such a situation by not making linearization assumptions.

Table 5 summarizes the root-mean-square deviation of the MEDLI-related parameters from the truth for the time period MEDLI data is available. Looking at the overall deviation, it is clear that the UKF has a smaller residual for all of the MEDLI-related parameters and would be the preferred type of statistical estimator based solely on this metric. However, the EKF residual is really not that far off from the UKF deviation (except for a few exceptions, such as Mach number deviation for the dispersed dataset). Of course, if the true uncertainties were known, then one could perform a similar comparison between the two estimators. However, in lieu of such information, it is hard to differentiate between the EKF and UKF.

Table 5. Root-mean-square deviation of the estimation from the truth when MEDLI data is available.

\begin{tabular}{lcccc}
\hline \multicolumn{1}{c}{ State } & EKF Nominal & UKF Nominal & EKF Dispersed & UKF Dispersed \\
\hline Angle of attack (deg.) & 0.192 & 0.145 & 0.570 & 0.334 \\
Sideslip angle (deg.) & 0.211 & 0.140 & 0.506 & 0.242 \\
Mach number & 0.190 & 0.073 & 1.379 & 0.393 \\
Dynamic pressure (Pa.) & 38.51 & 16.27 & 43.56 & 25.73 \\
Axial force coefficient & 0.017 & 0.006 & 0.022 & 0.018 \\
Normal force coefficient & 0.0015 & 0.0014 & 0.003 & 0.001 \\
\hline \hline
\end{tabular}

If the truth information for the states were not known, it seems that either method is acceptable for reconstruction. EKF will be expected to have divergence issues if the reconstruction is conducted over a long time period. UKF is computationally more time consuming since $2 n+1$ sigma points have to be propagated in time instead of just the propagation of the state vector in the EKF. However, if the truth is unknown, perhaps the best approach would be to use both estimators and then compare results. Both estimators had good performance in estimating trajectory, atmosphere and aerodynamics from the simulated MEDLI dataset and the same type of performance would be expected from the estimators for an unknown dataset.

\section{Future Work}

As mentioned earlier, both EKF and UKF provide a best estimate of the state and the uncertainty of the parameters of interest. Future work would involve doing a comparison between the estimated uncertainties with truth uncertainties generated by Monte Carlo analysis. Literature suggests the UKF should be better at the uncertainty quantification than the EKF. Thus, this comparison may help decide what type of estimator should be used with MEDLI-like data when the truth is unknown. Moreover, work is also needed to correlate the values of the 
process noise parameters and the estimation capability of the EKF and UKF. Guidelines could be developed to decide what process noise and tuning parameters to use when processing real-life data. Finally, a sensitivity study could be performed to gauge what type of data (IMU, MEDLI or radar altimeter) is important in the reconstruction of trajectory, atmospheric and aerodynamic parameters.

\section{Conclusions}

Measurements from the MEDLI suite will provide valuable data that can be used in EDL parameter reconstruction. With the MEADS data and a statistically-based reconstruction methodology shown here, one can simultaneously reconstruct a vehicle's trajectory, atmospheric parameters and aerodynamic coefficients. Due to the statistical nature of the estimator, this process also produces information about the uncertainty in the estimated parameters. Sample reconstruction results from two statistical estimators, EKF and UKF, were shown for two simulated Mars entry datasets. Generally, the two estimators reconstructed the data within the goals of the MEDLI science objectives. The largest errors were observed for estimation of the Mach number and normal force coefficient. The methodology demonstrated here can be applied to the MSL dataset that will become available in the latter part of 2012.

\section{Acknowledgments}

The Space Systems Design Laboratory at the Georgia Institute of Technology developed the tools used to generate the results in this paper. A NASA Research Announcement (NRA) award (No. NNX08AI18A) has supported some of the tool development effort. The authors would also like to thank Chris Karlgaard of Analytical Mechanics Associates and Mark Schoenenberger of NASA Langley Research Center for their advice regarding the simulation of the MEDLI-like data and the estimation of EDL parameters from this data.

\section{References}

1 Ingolby, R., Michel, F., Flaherty, Doty, T., M.G., Preston, B., Villyard, K., and Steele, R., "Entry Data Analysis for Viking Landers 1 and 2,” NASA CR-159388, 1976.

2 Spencer, D., Blanchard, R., Braun, R., Kallemeyn, P., and Thurman, S., "Mars Pathfinder Entry, Descent, and Landing Reconstruction," Journal of Spacecraft and Rockets, Vol. 36, No. 3, 1999, pp. 357-366.

3 Blanchard, R., "Entry Descent and Landing Trajectory and Atmosphere Reconstruction for the Mars Exploration Rovers Missions A and B," Tech. rep., White paper performed under NASA-JPL subcontract CCNS20568F, The George Washington University, 2008.

4 Desai, P.N., Prince, J.L., Queen, E.M., Schoenenberger M., Cruz, J.R., and Grover, M.R., "Entry, Descent, and Landing Performance of the Mars Phoenix Lander," Journal of Spacecraft and Rockets, Vol. 48, No. 5, 2011, pp. 798-808.

5 Blanchard, R.C., and Desai, P.N., "Mars Phoenix Entry, Descent, and Landing Trajectory and Atmosphere Reconstruction," Journal of Spacecraft and Rockets, Vol. 48, No. 5, 2011, pp. 809-821.

6 Withers, P., Towner, M., Hathi, B., and Zarneckbi, J., "Analysis of entry accelerometer data: A case study of Mars Path," Planetary and Space Science, Vol. 51, 2003, pp. 541-561.

7 Streipe, S., Way, D., Dwyer, A., and Balaram, J., "Mars Science Laboratory Simulations for Entry, Descent, and Landing," Journal of Spacecraft and Rockets, Vol. 43, No. 2, 2006, pp. 311-323.

8 Edquist, K. T., Desai, P. N., and Schoenenberger, M., "Aerodynamics for the Mars Phoenix Entry Capsule," AIAA/AAS Astrodynamics Specialist Conference and Exhibit, AIAA 2008-7219, Honolulu, HI , 2008.

9 Edquist, K. T., "Computations of Viking Lander Capsule Hypersonic Aerodynamics with Comparisons to Ground and Flight Data," AIAA Atmospheric Flight Mechanics Conference and Exhibit, AIAA 2006-6137, Keystone, CO, 2006.

10 Desai, P. N. and Knocke, P. C., "Mars Exploration Rovers Entry, Descent, and Landing Trajectory Analysis," AIAA/AAS Astrodynamics Specialist Conference and Exhibit, AIAA 2004-5092, Providence, RI , 2004.

11 Regan, F. J. and Anandakrishnan, S. M., Dynamics of Atmospheric Re-Entry, American Institute of Aeronautics and Astronautics, Inc., Reston, VA, 1993.

12 Euler, E., Adams, G., and Hopper, F., "Design and Reconstruction of the Viking Lander Descent Trajectories," Journal of Guidance and Control, Vol. 1, No. 5, 1978, pp. 372-378.

13 Schofield, J. T., Barnes, J. R., Crisp, D., Haberle, R. M., Larsen, S., Magalhaes, J. A., Murphy, J. R., and A. Seiff, G. W., “The Mars Pathfinder Atmospheric Structure Investigation/Meteorology (ASI/MET) Experiment," Science, Vol. 278, No. 5344, 1997, pp. 1752-1758.

14 Milos, F. S., Chen, Y., Congdon, W. M., and Thornton, J. M., "Mars Pathfinder Entry Temperature Data, Aerothermal Heating, and Heatshield Material Response," Journal of Spacecraft and Rockets, Vol. 36, No. 3, 1999, pp. 380-391.

15 Withers, P. and Smith, M. D., "Atmospheric entry profiles from the Mars Exploration Rovers Spirit and Opportunity," Icarus, Vol. 185, 2006, pp. 133-142.

16 Gazarik, M., Wright, M., Little, A., Cheatwood, F., Herath, J., Munk, M., Novak, J., and Martinez, E., "Overview of the MEDLI Project," IEEE Aerospace Conference, IEEEAC 1510, Big Sky, MT, 2008. 
17 Seiff, A. and Reese Jr., D., "Use of Entry Vehicle Response to Define the Properties of the Mars Atmosphere," American Astronautical Society Symposium on Unmanned Exploration of the Solar System, NASA-TM-X-56125, Denver CO, 1965.

18 Hopper, F., "Trajectory, Atmosphere, and Wind Reconstruction from Viking Measurements," AIAA/AAS Astrodynamics Conference, AAS 75-068, Nassau, Bahamas, 1975.

19 Blanchard, R. and Walberg, G., "Determination of the Hypersonic-Continuum/Rarefied-Flow Drag Coefficient of the Viking Lander Capsule 1 Aeroshell from Flight Data," NASA TP 1793, 1980.

20 Pruett, C., Wolf, H., Heck, M., and Siemers, P., "Innovative Air Data System for the Space Shuttle Orbiter," Journal of Spacecraft and Rockets, Vol. 20, No. 1, 1983, pp. 61-69.

21 Edquist, K. T., Dyakonov, A. A., Wright, M. J., and Tang, C. Y., "Aerothermodynamic Design of the Mars Science Laboratory Heatshield," 41st AIAA Thermophysics Conference, AIAA 2009-4075, San Antonio, TX, No. 2009.

22 Mahzari, M., Cozmuta, I., Clark, I.G., and Braun, R.D., "An Inverse Parameter Estimation Methodology for the Analysis of Aeroheating and Thermal Protection System Experimental Data," 42nd AIAA Thermophysics Conference, AIAA 2011-4027, Honolulu, HI.

23 Striepe, S.A., Powell, R.W., Desai, P.N., Queen, E.M., Brauer, G.L., Cornick, D.E., Olson, D.W., Peterson, F.M., Stevenson, R., Engel, M.C., Marsh, S.M., and Gromko, A.M., "Program To Optimize Simulated Trajectories (POST II): Utilization Manual," Vol. II, Version 1.1.6.G., 2004.

24 Christian, J., Verges, A., and Braun, R., "Statistical Reconstruction of Mars Entry, Descent, and Landing Trajectories and Atmospheric Profiles," AIAA SPACE Conference and Exposition, AIAA 2007-6192, Long Beach, CA, 2007.

25 Kalman, R., "A New Approach to Linear Filtering and Prediction Problems," ASME Journal of Basic Engineering, Vol. 82, 1960, pp. 35-45.

26 Dutta, S., Braun, R.D., "Mars Entry, Descent, and Landing Trajectory and Atmosphere Reconstruction," 48th AIAA Aerospace Sciences Meeting, AIAA 2010-1210, Orlando, FL, 2010.

27 Dutta, S., Clark, I.G., Russell, R.P., and Braun, R.D., "Statistical Entry, Descent and Landing Performance Reconstruction of the Mars Phoenix Lander," 8th International Planetary Probe Workshop, Portsmouth, VA, 2011.

28 Karlgaard, C.D., Beck, R.E., O’Keefe, S.A., Siemers, P.M., White, B.A., Engelund, W.C., and Munk, M.M., "Mars Entry Atmospheric Data System Modeling and Algorithm Development," 41st AIAA Thermophysics Conference, AIAA 2009-3916, 2009.

29 Wells, G.W. and Braun, R.D., "Reconstruction of the Spirit Mars Exploration Rover Entry, Descent and Landing Performance,", 2nd International ARA Days Conference, AA-3-2008-16, Arachon, France, 2008.

30 Wells, G., "A Comparison of Multiple Techniques for the Reconstruction of Entry, Descent, and Landing Trajectories and Atmospheres," Ph.D. Dissertation, Department of Aerospace Engineering, Georgia Institute of Technology, Atlanta, GA, 2011 .

31 Julier, S., Uhlmann, J., and Durrant-Whyte, H., "A New Method for the Nonlinear Transformation of Means and Covariances in Filters and Estimators," IEEE Transactions on Automatic Control, Vol. 45, No. 3, 2000, pp. 477-482.

32 Vinh, N.X., Busemann, A., and Culp, R.D., Hypersonic and Planetary Entry Flight Mechanics, The University of Michigan Press, Ann Arbor, MI, 1980.

33 Etkin, B., Dynamics of Atmospheric Flight, Dover Publications, Inc., Meneola, NY, 2000.

34 Kuipers, J., Quaternions and Rotation Sequences, Princeton University Press, Princeton, NJ, 1999.

35 Jaswinski, A., Stochastic Processes and Filtering Theory, Academic Press, San Diego, CA, 1970.

36 Tapley, B.D., S. B. and Born, G., Statistical Orbit Determination, Elsevier Academic Press, Burlington, MA, 2004.

37 Zarchan, P. and Musoff, H., Fundamental of Kalman Filtering, A Practical Approach, American Institute of Aeronautics and Astronautics, Inc., Reston, VA, 2000.

38 Simon, D., Optimal State Estimation, Wiley-Interscience Inc., Hoboken, New Jersey, 2006.

39 Lee, D., and Alfriend, K.T., "Sigma Point Filtering for Sequential Orbit Estimation and Prediction," Journal of Spacecraft and Rockets, Vol. 44, No. 2, 2007, pp. 388-398.

40 Fraser, D. and Potter, J., "The Optimum Linear Smoothers as a Combination of Two Optimum Linear Filters," IEEE Transactions on Automatic Control, Vol. 14, No. 8, 1969, pp. 387-390. 